\title{
Idle Channel Time Estimation in Multi-hop Wireless Networks
}

\author{
Simon Odou, Steven Martin, Khaldoun Al Agha \\ LRI - Université Paris-Sud - CNRS \\ 91405 Orsay, France \\ \{simon.odou,steven.martin,khaldoun.alagha\}@lri.fr
}

\begin{abstract}
This paper presents a theoretical estimation for idle channel time in a multi-hop environment. Idle channel time is the time proportion of a node during which the channel state is idle. Thus, it can be used to evaluate the available bandwidth. Major related work considers a cross-layer model, where the idle channel time measure is available at the MAC layer, but it is rarely implemented. Furthermore, this measure is not very flexible: it cannot differentiate the traffic's priorities and it works under the hypothesis that flows are strictly policed. Estimating instead of measuring the idle channel time prevents these drawbacks. This estimation is computed in three steps by (1) calculating the idle channel time bounds, (2) evaluating the probability of a given idle channel time value, (3) computing the expected value of this distribution and deducing the average idle channel time. We show by simulation that our estimation is accurate.
\end{abstract}

\section{INTRODUCTION}

A multi-hop wireless network is a network where each node is able to join others transparently. In such networks, two distant nodes cannot communicate directly and use intermediate nodes to relay messages. A node can successfully communicate with another node within its transmission range. In this case, the channel state is "transmitting" for the emitting node and "receiving" for the other one. A node outside this range but close enough to detect the signal would have a "noisy" channel state. Receivers or noisy channel nodes are in the emitter's carrier sensing range. The channel state is considered idle if no transmission (or noise) exists.

Idle channel time gives an accurate available bandwidth estimation since it represents the channel state. Therefore it accounts for interference among flows (inter-flow contention) and interference between a flow and itself (intra-flow contention). This is the reason why the idle channel time measure is widely used in admission control mechanisms which aim at accepting a new flow only if it does not degrade the quality of service of existing flows with higher or equal priority.

In this paper, we present a theoretical estimation for a node's idle channel time. We assume that the flows within its carrier sensing range are known.

Related work based on the idle channel time measure has several drawbacks. Estimating instead of measuring idle channel time prevents the following problems:

- Previous work assumes the idle channel time measure is retrievable from the MAC layer. Therefore a cross-

This work was supported by a grant from DGA (French Army Research Dept). layer model is necessary. But such models are scarce in practice. Estimating idle channel time removes this need.

- In the literature, flows are assumed to be well policed so that they consume exactly the bandwidth they have requested. This hypothesis is not realistic since subconsumption may occur. In this case, the channel would spend more time in the idle state and it would give a wrong available bandwidth estimation. As our estimation is based on reservations, sub-consumption has no impact.

- Prior work does not support quality of service. For example, if the remaining available bandwidth is used for best-effort traffic, the idle channel time measure is not useful. But, our approach computes the estimation for a set of flows. Therefore it is possible to compute the idle channel time estimation for a given priority level.

- Related work does not predict the impact of a new flow on the available bandwidth: the idle channel time measure does not give an information about intra-flow contention. In our solution we can perform the estimation for a set of flows including this new flow. Thus, it predicts directly the available bandwidth as if the new flow was already in the network.

The idle channel time estimation problem is not trivial in multi-hop wireless networks. A node $n$ can have within its carrier sensing range two nodes not interfering with each other. In this case, if these two nodes transmit at the same time, their transmissions appear on the channel of $n$ as a superposition of two signals. This phenomenon is called "spatial reuse". To begin, we study the idle channel time bounds: the lower and upper bounds correspond respectively to the minimum and maximum spatial reuse cases. Then, we compute the probability for intermediary cases. From this distribution, we deduce the expected idle channel time value. Finally, we evaluate by simulation the accuracy of our estimation.

The paper is organized as follows. Related work is discussed in section 2. Section 3 is used to describe our idle channel time estimation. Simulations results are presented in section 4, before we conclude the paper.

\section{RELATED WORK}

The channel state has been largely studied in the literature in particular in a single hop environment with a 802.11 MAC layer [1]. But to our knowledge, no existing work has focused on the idle channel time estimation problem (i) in a multi-hop 
environment and (ii) from a general point of view (i.e. for any slotted random access MAC layer). So we present works based on the idle channel time measure such as available-bandwidth estimators. An efficient available-bandwidth estimator considers the following factors: intra/inter-flows contention, spatial reuse and sublayer wastage. We review algorithms that estimate the available bandwidth when priorities of flows are considered and when they are not.

\section{A. Available bandwidth estimation without priorities on flows}

The estimation of the available bandwidth using the idle channel time is straightforward. This is done in the "listen" bandwidth estimation [2] or in the CACP protocol [3]. The local available bandwidth of a node is $i t \cdot c / \Delta t$ where $i t$ is the time spent in the idle state during the listening duration $\Delta t$ and $c$ represents the channel capacity. The problem is then to decide if the local available bandwidth is enough to accept a new flow. Because of intra-flow contention, a node needs to allocate several times the bandwidth requested by a new flow. The number of times depends on the number of nodes of the path's flow within its carrier sensing range.

To consider the wastage due to the IEEE 802.11 MAC layer, VMAC [4] passively monitors the medium and emulates its behavior. Virtual data is generated and the virtual MAC tries to send it virtually. If at the same time, a packet transmission on the real MAC layer happens, the virtual MAC considers it as a collision and enters the backoff procedure.

These approaches are based on the channel sensing by a node. They have the following problems: a cross-layer model is needed; the sub-consumption problem is not addressed; the impact of a new flow is not precisely evaluated.

\section{B. Available bandwidth estimation with priorities on flows}

In previous work, the priorities of flows are not considered, even real-time and best-effort traffic are not distinguishable. We now focus on work estimating the available bandwidth in the case where flows are prioritized.

In [5], to limit the impact of a new flow, this one starts directly and is dismissed as soon as it begins to degrade the throughput of higher priority flows. This approach does not predict the impact of a new flow on the available bandwidth. So flows that are finally refused have needlessly degraded the throughput. Furthermore, it requires a cross-layer model.

Another approach is to compute the available bandwidth based on the reservations as in SWAN [6] or in the "hello" bandwidth estimation [2]. A node's available-bandwidth for a given priority is its capacity minus the sum of the reserved bandwidth of higher or equal priority for each node in its carrier sensing range. This approach considers the worst topology that is without spatial reuse. Thus, it gives an available bandwidth estimation which is actually the lower bound of what is achievable.

To synthesize, related work based on the channel listening approach considers the spatial reuse factor but needs a crosslayer model and cannot differentiate the traffic. Other related work based on the reservations approach can differentiate the traffic but do not consider spatial reuse.

\section{SOLUTION}

In this paper, we present a theoretical estimation of a node's idle channel time. We assume that flows within its carrier sensing range are known. This solution is interesting because:

- it does not use a cross-layer model;

- it can distinguish the traffic of a particular flow set;

- it is not misled by sub-consummation of flows;

- it can predict the impact of a new flow, including the intra-flow contention effects.

Because of spatial reusing, two nodes in the carrier sensing range of any node $n$ may be able to transmit at the same time. In this case, node $n$ "sees" the two signals summed on its channel but it has no way to determine how many transmissions are summed, it just knows that its channel is busy ${ }^{1}$. Therefore, the idle channel time of node $n$ depends on spatial reuse.

We first present the network model we consider. Then we define the lower (resp. upper) bound of idle channel time, that is the value obtained when the nodes which are able to transmit simultaneously do it as often as possible (resp. not at all). We compute the probability to be in each possible point of this interval, the idle channel time estimation being the expected value of the distribution.

\section{A. Network model}

The classical representation of a network is a graph where vertexes are nodes and edges are transmission links. A link $(s, d)$ is a pair of nodes where $d$ is in the transmission range of $s$. A network topology is a directed graph $G=(V, E)$ where $V$ is a set of nodes and $E$ a set of links. For example, Figure 1 represents a topology of five nodes ${ }^{2}$.

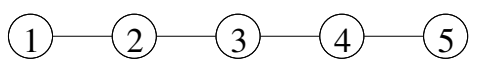

Fig. 1. Network topology

The set of nodes interfering with node $n$ is denoted $I_{n}$. It can be estimated by considering:

- power control where $n$ can emit with a higher power to contact nodes within its carrier sensing range directly [3];

- a N-hop interference model where node $n$ interferes with its N-hop neighbors;

- localization informations [7].

The whole network interferences can be represented by a conflict graph where interfering links are linked. It is built from the knowledge of the local interferences $I_{n}, \forall n \in V$. The conflict graph of a topology is an undirected graph $C G=\left(V^{\prime}, E^{\prime}\right)$ where $V^{\prime}=E$ is the set of links and where edges belonging to $E^{\prime}$ join interfering links. Let assume, for example, a 2-hop interference model, where a node interferes with its neighbors and the neighbors of its neighbors. Then, we obtain the conflict graph of Figure 2 from the topology of

\footnotetext{
${ }^{1}$ In the rest of this article, we consider that the channel is busy when its state is receiving, transmitting or noisy.

${ }^{2}$ To simplify our example, the graph is undirected.
} 
Figure 1. As nodes 2 and 3 are in the carrier sensing range of node 1 , link $(1,2)$ is interfering with links $(2,3)$ and $(3,4)$.

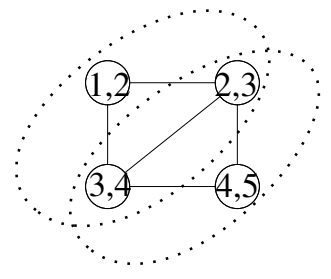

Fig. 2. Conflict graph

The spatial reuse can be represented by grouping either interfering links with each other (clique) or not interfering links (independent set). A clique $C$ of $C G$ is a set of links interfering with each other. $\mathcal{C}$ is the set of the maximal cliques of a conflict graph $C G$. A maximal clique is a clique where it is impossible to add another link. For example, the maximal cliques of the conflict graph on Figure 2 are: $\{(1,2)(2,3)(3,4)\}$ and $\{(2,3)(3,4)(4,5)\}$. Determining the cliques of a graph is NP-complete but polynomial heuristics exist [8]. The independent sets problem is equivalent [9], [10]. But actually it is easier to compute cliques since they are made of closed links and nodes usually have a better knowledge of their neighborhood. So cliques are often preferred in the literature to compute constraints on flows [7], [11].

We focus on the idle channel time of a given node $n$. Let $\mathcal{C}_{n}$ be the local clique view of $n$. More precisely, $\mathcal{C}_{n}$ contains the cliques of $\mathcal{C}$ where the links for which the source emissions have no impact on the channel of $n$ are removed:

$$
\mathcal{C}_{n}=\bigcup_{C \in \mathcal{C}}\left\{\left\{(s, d) \mid(s, d) \in C \wedge s \in\{n\} \cup I_{n}\right\}\right\} \backslash\{\emptyset\}
$$

For example, on Figure $2, \mathcal{C}_{1}$ contains $\{(2,3)(3,4)\}$ and $\{(1,2)(2,3)(3,4)\}$. We also define $L_{n}$ as the set of links for which node $n$ is in the source carrier sensing range: $L_{n}=\bigcup_{C \in \mathcal{C}_{n}} C$. Following the previous example, $L_{1}=$ $\{(1,2)(2,3)(3,4)\}$.

We consider that time is slotted and we define $t_{s}$ as the slot duration. The idle channel time estimation is computed for a given duration $n_{s} \cdot t_{s}$ where $n_{s} \in \mathbb{N}^{*}$ is the number of slots. For the sake of simplicity, we assume that all the links of $E$ have the same capacity $c$ and that the packet size $\sigma$ is constant. So the transmission delay of a packet is $t_{p}=\frac{\sigma}{c}$. The reserved bitrate on link $l$ is denoted $u_{l}$. The number of packets on link $l$ during $n_{s} \cdot t_{s}$ is $n_{p, l}=n_{s} \cdot t_{s} \cdot \frac{u_{l}}{\sigma}$.

\section{B. Idle channel time bounds}

We are now interested in bounding the idle channel time. By definition, among the links of a clique, only one can transmit at a time, contrary to links belonging to different cliques. Thus, the maximum number of simultaneous transmissions summed on the channel of $n$ is equal to $\left|\mathcal{C}_{n}\right|$.

$M_{n}$, the maximum number of busy slots on the channel of node $n$ during $n_{s} \cdot t_{s}$, is equal to the sum of the slots consumed by each link of $L_{n}$ :

$$
M_{n}=\min \left(n_{s} ;\left\lfloor\frac{t_{p}}{t_{s}} \cdot \sum_{l \in L_{n}} n_{p, l}\right\rfloor\right) .
$$

$N_{n}$, the minimum number of busy slots on the channel of node $n$ during $n_{s} \cdot t_{s}$, is equal to the number of slots consumed by the clique having the highest reserved bandwidth:

$$
N_{n}=\max _{C \in \mathcal{C}_{n}}\left(\left\lceil\frac{t_{p}}{t_{s}} \cdot \sum_{l \in C} n_{p, l}\right\rceil\right) .
$$

By Equations 1 and 2, it derives that the lower and upper bounds on the idle channel time of node $n$ during $n_{s}$ slots are equal to: $i c t m i n_{n}=1-M_{n} / n_{s}$ and $i c t \max _{n}=1-N_{n} / n_{s}$.

\section{Spatial reuse probability}

We are now interested in computing $\widetilde{i c t}_{n}$, the estimation of

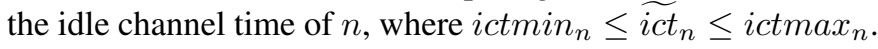

We now assume that a node transmits a whole packet in exactly one slot $\left(t_{s}=t_{p}\right)$ and that nodes are synchronized. Let $X_{n}$ be the discrete random variable giving the number of busy slots on the channel of $n$ among the $n_{s}$ slots considered and $\operatorname{Pr}\left(X_{n}=x\right)$ the associated probability. Thus, $\operatorname{Pr}\left(X_{n}=x\right)$ is equal to the number of possibilities to have $x$ busy slots on the channel of $n$ among the $n_{s}$ slots considered, divided by the total number of possibilities. We compute this probability in the following subsection, proceeding in two steps:

- determination of the number of possibilities, for each node, to transmit its packets in at most $x$ slots;

- determination of the number of possibilities, for each node, to transmit its packets in exactly $x$ slots.

Finally, we propose an equivalent non-recursive form for this computation.

1) Transmission of packets in at most $x$ slots:

Let $g_{n}(x)$ be the total number of possibilities to transmit the packets of the links belonging to $L_{n}$ in at most $x$ slots when links are not interfering. Thus, $g_{n}(x)$ is equal to the number of possibilities for a first link $l_{1}$ to have its $n_{p, l_{1}}$ packets transmitted in at most $x$ slots, times the number of possibilities for a second link $l_{2}$ to have its $n_{p, l_{2}}$ packets transmitted in at most $x$ slots, and so on. Hence, $g_{n}(x)$ is a product of combinations defined for $x \in\left[N_{n}, M_{n}\right]$ :

$$
g_{n}(x)=\prod_{i=1}^{\left|L_{n}\right|}\left(\begin{array}{c}
x \\
n_{p, l_{i}}
\end{array}\right)=\prod_{i=1}^{\left|L_{n}\right|} \frac{x !}{n_{p, l_{i}} ! \cdot\left(x-n_{p, l_{i}}\right) !} .
$$

Let $g_{n}^{\prime}(x)$ be the generalization of $g_{n}(x)$, when links are interfering. In this case, a slot consumed by any link $l \in L_{n}$ is no more available for the other links interfering with $l$.

$$
g_{n}^{\prime}(x)=\prod_{i=1}^{\left|L_{n}\right|}\left(\sum_{\substack{j=1 \\\left\{l_{i}, l_{j}\right\} \in E^{\prime} \\ n_{p, l_{i}}}}^{i-1} n_{p, l_{j}}\right)
$$


2) Transmission of packets in exactly $x$ slots:

We now consider the number of possibilities to have exactly $x$ busy slots on the channel of node $n$ among $n_{s}$ slots when links belonging to $L_{n}$ are transmitting. This function, $f_{n}(x)$, is defined recursively for $x \in\left[N_{n}, M_{n}\right]$. Indeed, it is equal to (i) the number of possibilities to have at most $x$ busy slots among $n_{s}$ slots, minus (ii) the number of possibilities to transmit them in exactly $x-1$ slots times the number of possibilities to choose $x-1$ slots among $x$, minus (iii) the number of possibilities to transmit them in exactly $x-2$ slots times the number of possibilities to choose $x-2$ slots among $x$, and so on for $x-3, x-4, . ., N_{n}$. The first term is previously defined by $g_{n}^{\prime}(x)$. The second, third and following terms are a product of a combination and a recursive call to $f$. So $f_{n}(x)$ is equal to:

$$
\begin{cases}f_{n}(x)=g_{n}^{\prime}(x) & \text { if } x=N_{n} \\
f_{n}(x)=g_{n}^{\prime}(x)-\sum_{i=N_{n}}^{x-1}\left(\begin{array}{l}
x \\
i
\end{array}\right) f_{n}(i) & \text { if } \left.x \in] N_{n}, M_{n}\right]\end{cases}
$$

It is clear that $\operatorname{Pr}\left(X_{n}=x\right)$, the probability to have $x$ busy slots on the channel of $n$ among the $n_{s}$ slots considered, is null when $x<N_{n}$ or $x>M_{n}$. Then, we obtain from Equations 4 and 5 the following property:

$$
\begin{cases}\operatorname{Pr}\left(X_{n}=x\right)=\frac{\left(\begin{array}{c}
n_{s} \\
x
\end{array}\right) \cdot f_{n}(x)}{g_{n}^{\prime}\left(n_{s}\right)} & \text { if } x \in\left[N_{n}, M_{n}\right] \\
\operatorname{Pr}\left(X_{n}=x\right)=0 & \text { otherwise. }\end{cases}
$$

We show that $\operatorname{Pr}\left(X_{n}=x\right)$ is a probability law. Indeed:

$$
\begin{aligned}
\sum_{x \in \mathbb{N}} \operatorname{Pr}\left(X_{n}=x\right) & =\frac{\sum_{i=N_{n}}^{n_{s}}\left(\begin{array}{c}
n_{s} \\
i
\end{array}\right) f_{n}(i)}{g_{n}^{\prime}\left(n_{s}\right)} \\
& =\frac{\sum_{i=N_{n}}^{n_{s}-1}\left(\begin{array}{c}
n_{s} \\
i
\end{array}\right) f_{n}(i)+f_{n}\left(n_{s}\right)}{g_{n}^{\prime}\left(n_{s}\right)} \\
& =\frac{\sum_{i=N_{n}}^{n_{s}-1}\left(\begin{array}{c}
n_{s} \\
i
\end{array}\right) f_{n}(i)+g_{n}^{\prime}\left(n_{s}\right)-\sum_{i=N_{n}}^{n_{s}-1}\left(\begin{array}{c}
n_{s} \\
i
\end{array}\right) f_{n}(i)}{g_{n}^{\prime}\left(n_{s}\right)} \\
& =1 .
\end{aligned}
$$

3) Non-recursive equivalent form:

Let $h_{n}(x)$ be an equivalent non-recursive form of $f_{n}(x)$ defined as follows for $x \in\left[N_{n}, M_{n}\right]$ :

$$
h_{n}(x)=\sum_{j=N_{n}}^{x}(-1)^{j+N_{n}}\left(\begin{array}{c}
x \\
j-N_{n}
\end{array}\right) g_{n}^{\prime}\left(x+N_{n}-j\right) .
$$

Thus we have the equivalence property:

$$
\forall x \in\left[N_{n}, M_{n}\right], \quad f(x)=h(x) .
$$

Proof: By recurrence. Equation 6 is satisfied for $x=N_{n}$, as $h_{n}\left(N_{n}\right)=g_{n}^{\prime}\left(N_{n}\right)=f_{n}\left(N_{n}\right)$. Assuming that the equation is true for $x \leq k$, that is $\forall i \in\left[N_{n}, k\right], h_{n}(i)=f_{n}(i)$, we get:

$$
\begin{aligned}
f_{n}(k+1) & =g_{n}^{\prime}(k+1)-\sum_{i=N_{n}}^{k}\left(\begin{array}{c}
k+1 \\
i
\end{array}\right) f_{n}(i) \\
& =g_{n}^{\prime}(k+1)-\sum_{i=N_{n}}^{k}\left(\begin{array}{c}
k+1 \\
i
\end{array}\right) h_{n}(i)
\end{aligned}
$$

Then we focus on the sum for $i=N_{n} . . k$ of the previous equation. By expanding $h_{n}(i)$, it becomes:

$$
\sum_{i=N_{n}}^{k}\left(\begin{array}{c}
k+1 \\
i
\end{array}\right) \sum_{j=N_{n}}^{i}(-1)^{j+N_{n}}\left(\begin{array}{c}
i \\
j-N_{n}
\end{array}\right) g_{n}^{\prime}\left(i+N_{n}-j\right) .
$$

The last term of the inner sum is the only one which contains $g_{n}^{\prime}\left(N_{n}\right)$. Thus, we extract and factorize it. This last term is:

$$
\begin{aligned}
& \sum_{i=N_{n}}^{k}\left(\begin{array}{c}
k+1 \\
i
\end{array}\right)(-1)^{i+N_{n}}\left(\begin{array}{c}
i \\
i-N_{n}
\end{array}\right) g_{n}^{\prime}\left(N_{n}\right) \\
& =g_{n}^{\prime}\left(N_{n}\right) \cdot \sum_{i=N_{n}}^{k}\left(\begin{array}{c}
k+1 \\
i
\end{array}\right)(-1)^{i+N_{n}}\left(\begin{array}{c}
i \\
i-N_{n}
\end{array}\right) \\
& =g_{n}^{\prime}\left(N_{n}\right) \cdot(-1)^{k+N_{n}} \cdot\left(\begin{array}{c}
k+1 \\
k-N_{n}+1
\end{array}\right)
\end{aligned}
$$

In the same way, we extract the last remaining term of the inner sum of Equation 8 and we successively factorize by $g_{n}^{\prime}\left(N_{n}+1\right), . ., g_{n}^{\prime}(k-1), g_{n}^{\prime}(k)$. The sum of these factorizations, equivalent to Equation 8, is:

$$
\sum_{j=N_{n}}^{k}(-1)^{j+N_{n}} g_{n}^{\prime}\left(k+N_{n}-j\right)\left(\begin{array}{c}
k+1 \\
j-N_{n}+1
\end{array}\right)
$$

Then we increment the indexes of this resulting sum in order to insert the term $g_{n}^{\prime}(k+1)$ of Equation 7. So we get:

$$
-\sum_{j=N_{n}+1}^{k+1}(-1)^{j+N_{n}} g_{n}^{\prime}\left(k+1+N_{n}-j\right)\left(\begin{array}{c}
k+1 \\
j-N_{n}
\end{array}\right)
$$

From Equation 7, we obtain $g_{n}^{\prime}(k+1)$ minus the previous sum equal to $h_{n}(k+1)$.

This non recursive function can be implemented very efficiently by using the $\Gamma$ function instead of factorials.

\section{Idle channel time estimation}

We determine in previous subsections the probability $\operatorname{Pr}\left(X_{n}=x\right)$ to have, on the channel of node $n$, exactly $x$ busy slots during the interval $n_{s} \cdot t_{s}$. Hence, we obtain the estimation of the idle channel time of node $n$ by computing the expected value of the random variable $X_{n}$, that is:

$$
\widetilde{i c t}_{n}=1-\frac{E\left(X_{n}\right)}{n_{s}}=1-\frac{\sum_{i=0}^{n_{s}} i \cdot \operatorname{Pr}\left(X_{n}=i\right)}{n_{s}} \text {. }
$$

\section{RESUlTS}

We compare by means of simulations our estimation and the measure of idle channel time, with and without collisions. We have implemented these algorithms on a IEEE 802.11b MAC layer under Opnet Modeler with the following parameters: $\sigma=1500$ bytes, $t_{s}=2.5 \mathrm{~ms}, n_{s}=400$ slots.

First, we have simulated a collision-free network where the transmitting links are distant enough to avoid interference. Moreover, a specific node $n$ is in the carrier sensing range of all other nodes. Then, we measure and estimate the idle channel time of this node. Figures 3 and 4 represent the measure, the estimation and the bounds on its idle channel time where the number of nodes is respectively 11 and 61, and where the number of transmitting links is respectively 5 and 30. Graphs are plotted for a total traffic varying from 0 to the maximum capacity, fairly shared among links. We notice that our estimation of the idle channel time is accurate. Indeed, the error does not exceed 3\%. This difference mainly comes from rounding values used in our computation.

Then we have simulated a network composed by 5 nodes where there exists interference among links, thus, collisions may occur. Figure 5 represents the measure, the estimation and the bounds on the idle channel time of node $n$, that is the node in the carrier sensing range of the others. As in the previous simulations, the total traffic varies from 0 to the 
maximum capacity. The more the traffic grows, the less the channel of node $n$ is idle. In theory, the channel of $n$ is always busy once the traffic has reached the maximum capacity. In practice, even if the traffic is maximum, the channel of $n$ stays idle a small part of time due to the backoff algorithm which addresses the medium access problem. This can be observed on Figure 5, where the idle channel time measure exceeds the upper bound when the total traffic is maximum. This is due to the fact that we have adopted a general network model, without consideration to a given MAC layer. The specificities of a MAC layer can be easily integrated in our model.

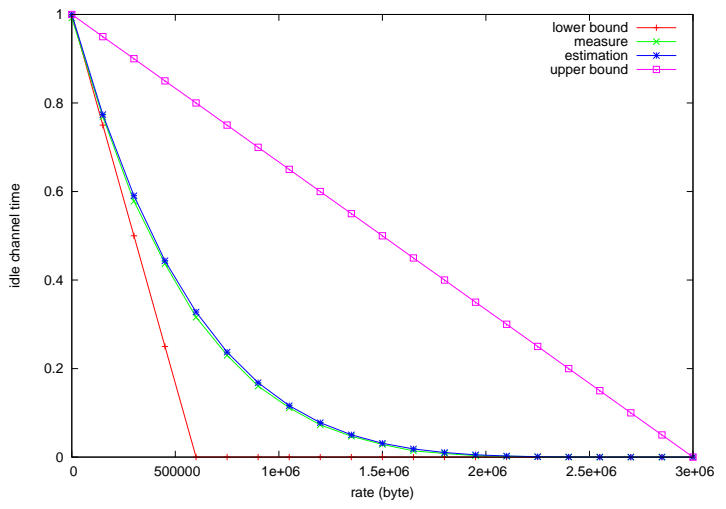

Fig. 3. Idle channel time for a network of 11 nodes without collision

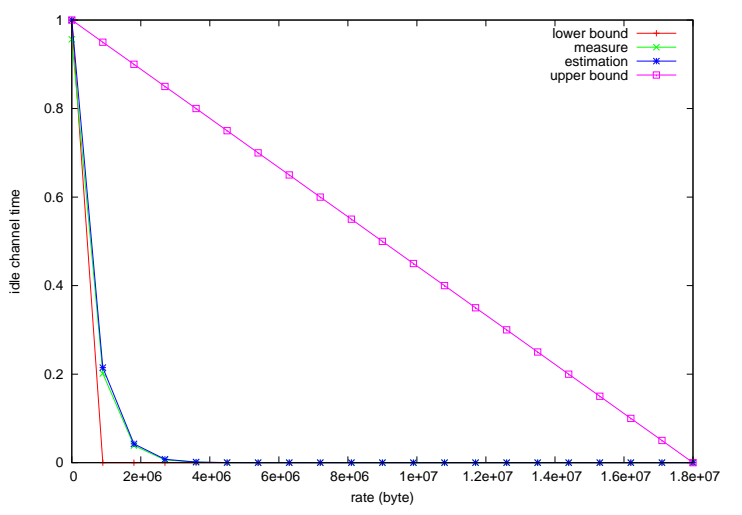

Fig. 4. Idle channel time for a network of 61 nodes without collision

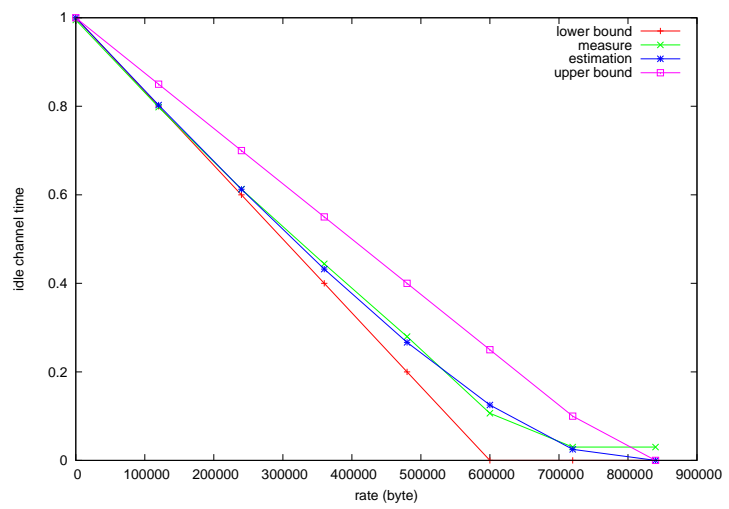

Fig. 5. Idle channel time for a network of 5 nodes with collisions

\section{CONCLUSion}

In this paper, we have studied the problem of estimating the idle channel time of a node in a multi-hop environment (i.e. with concurrent transmissions) and from a general point of view (i.e. for any slotted random access MAC layer). Our main contribution is a probabilistic model which computes a node's idle-channel-time estimation based on the knowledge of the flows reservations within its radio range.

First we have lower and upper bounded the idle channel time depending whether the links that can transmit simultaneously do it or not. Then we have computed the probability for a given idle channel time value. Finally, we have estimated the idle channel time from the expected value of this distribution. Simulations have shown that this estimation is precise for a 802.11b MAC layer.

The idle channel time is largely used because it gives an accurate available bandwidth estimation. Estimating instead of measuring its value has several advantages: no cross-layer model is needed, flows can have different priorities, strict flows policing is not necessary, the prediction of a new flow's impact on the network is much more precise. Then related work on admission control would benefit to use an available-bandwidth predictor based on the idle channel time estimation.

In the future, it would be interesting to apply our approach to more specific problems such as admission control in 802.11 ad hoc networks.

\section{REFERENCES}

[1] G. Bianchi, "Performance analysis of the IEEE 802.11 distributed coordination function," IEEE Journal on Selected Areas in Communications, vol. 18 , no. 3, 2000.

[2] L. Chen and W. B. Heinzelman, "Qos-aware routing based on bandwidth estimation for mobile ad hoc networks," IEEE journal on Selected Areas in Communications, vol. 23, no. 3, pp. 561-572, 2005.

[3] Y. Yang and R. Kravets, "Contention-aware admission control for ad hoc networks," IEEE Transactions on Mobile Computing, vol. 4, no. 4, pp. 363-377, 2005.

[4] M. Barry, A. T. Campbell, and A. Veres, "Distributed control algorithms for service differentiation in wireless packet networks," in INFOCOM 2001, vol. 1, 2001, pp. 582-590 vol.1.

[5] Y. Yang and R. Kravets, "Distributed qos guarantees for realtime traffic in ad hoc networks," in SECON 2004, 2004, pp. 118-127.

[6] G.-S. Ahn, A. T. Campbell, A. Veres, and L.-H. Sun, "Swan: service differentiation in stateless wireless ad hoc networks," in INFOCOM 2002, vol. 2, 2002, pp. 457-466 vol.2.

[7] R. Gupta, J. Musacchio, and J. Walrand, "Sufficient rate constraints for QoS flows in ad-hoc networks," Ad Hoc Networks, vol. 5, no. 4, pp. 429-443, May 2007.

[8] C. Bron and J. Kerbosch, "Algorithm 457: finding all cliques of an undirected graph," Commun. ACM, vol. 16, no. 9, pp. 575-577, September 1973.

[9] K. Jain, J. Padhye, V. Padmanabhan, and L. Qiu, "Impact of interference on multi-hop wireless network performance," Wireless Networks, vol. 11, no. 4, pp. 471-487, July 2005.

[10] H. Luo, S. Lu, and V. Bharghavan, "A new model for packet scheduling in multihop wireless networks," in MobiCom 2000, 2000, pp. 76-86.

[11] R. Gupta, Z. Jia, T. Tung, and J. Walrand, "Interference-aware QoS routing (IQRouting) for ad-hoc networks,” vol. 5, 2005, pp. 6 pp.+. 